\title{
La necesidad de definir un modelo de alfabetización en información para el Plan Ceibal
}

\author{
The need to define a model of information \\ literacy for the Plan Ceibal
}

\author{
María Gladys CERETTA' \\ Paola PICCO' (in memoriam)
}

\section{Resumen}

En este trabajo presentan los resultados preliminares de una investigación en curso que se viene realizando en el marco del Programa de Desarrollo Académico de la Información y la Comunicación de la Universidad de la República. La misma toma como objeto de estudio el Plan Ceibal, política pública de inclusión digital que está implementando el gobierno uruguayo. Dicho Plan ha otorgado un computador personal a cada niño de Escuelas Públicas a nivel nacional. La investigación pretende contribuir con dicho Plan, a través de la formulación de pautas para un Modelo de Alfabetización en Información que permita a los niños obtener las competencias necesarias para ser usuarios de la información críticos y responsables. Se mencionan las técnicas metodológicas utilizadas y se brinda la interpretación de los primeros resultados obtenidos. Se concluye que el Plan Ceibal no ha contemplado un modelo de alfabetización en información. Se reafirma la necesidad de contar con dicho modelo y se hace referencia a los componentes que deberían ser considerados por el mismo. Se aspira a generar un modelo integral de alfabetización en información para el Plan Ceibal que pueda ser extrapolado a otras áreas educativas del País.

Palabras-clave: Alfabetización digital. Alfabetización en información. Competencias en información. Modelo de alfabetización en información. Plan Ceibal.

\begin{abstract}
This paper presents the preliminary results of a research that is being carried our Program for Scholar Development of Information and Communication of Universidad de la República. The research focused on the Ceibal Program, a public policy for digital inclusion of Uruguayan children, implemented by the Uruguayan government. Through this program, every Uruguayan child attending public schools receives a laptop (one laptop per child program). The aim of our study was to develop guidelines for a Literacy Information Program that enables children to have the necessary competencies to be critical and responsible information users. It addressed the methodology used to develop the guidelines and discuss the preliminary results. It concluded that the Ceibal Program does not include a program for information literacy. It reaffirm the need for an information literacy program and point out the components that should be included in it. The aim is to create a comprehensive model for information literacy for the Ceibal Program that could be used by other educational areas in the country.
\end{abstract}

Keywords: Digital literacy. Information literacy. Information competencies. Information literacy program. Ceibal Program.

\section{Introducción}

El Plan Ceibal es el modelo uruguayo de una laptop por niño, las conocidas computadoras producidas por la organización One Laptop per Child (OLPC). Dicho Plan ha suscitado particular atención a nivel internacional porque es la primera experiencia de alcance nacional (Warschauer; Ames, 2010). A través del mismo, todos los

\footnotetext{
1 Profesoras, Universidad de la República, Escuela Universitaria de Bibliotecología y Ciencias Afines, Programa de Desarrollo Académico de la Información y la Comunicación. R. Emilio Frugoni, 1427, 11200, Montevideo, Uruguay. Correspondencia a nombre de/Correspondence to: M.G. CERETTA. E-mail:<mgceretta@gmail.com>.

Recibido el día 21/5/2012, re-presentado el 26/11/2012 y aceptado para su publicación el 6/12/2012.
} 
niños del Sistema Educativo Uruguayo han recibido su computadora personal y la utilizan en la clase y en sus hogares para realizar distintas tareas, ya sean educativas como de esparcimiento. La experiencia de entrega de computadores fue acompañada de la instalación de conexión inalámbrica a Internet en las escuelas y en espacios públicos. Se estimaba que el $95 \%$ de las escuelas tenía este tipo de conexión en el año 2011 y 273 lugares públicos permitían el acceso a Internet (Pittaluga, 2010).

Sin embargo, y a pesar de la relevancia del Plan a nivel nacional, el mismo no tuvo desde un principio, el impacto esperado, lográndose una baja apropiación de la tecnología y su utilización en el aula (Martínez et al., 2009; Administración Nacional de Educación Publica, 2011).

El Plan Ceibal surge a iniciativa del Poder Ejecutivo en el año 2007 y es implementado a partir de un decreto presidencial. Nace con todo el apoyo político pero carente del involucramiento de los principales actores, generando fuertes resistencia en los ámbitos educativos. Este aspecto se refleja con fuerza en la estructura propia que se crea para el Plan que funciona en forma paralela e independiente del Sistema Educativo Nacional, situación que paulatinamente se va modificando.

En sus objetivos primarios el Plan aspiraba a promover la inclusión digital buscando a largo plazo alcanzar la justicia social mediante la promoción de la igualdad en el acceso a la información. Sin embargo, ya desde su formulación establece que la sola inclusión de la tecnología no es suficiente, y que la iniciativa debe ir acompañada de una propuesta educativa acorde (Plan Ceibal, 2010).

Por otro lado, se viene dando una rica discusión a nivel internacional entre dos conceptos muy cercanos al Plan Ceibal: la alfabetización digital y la alfabetización en información (Badwen, 2002; Garcia-Quismondo, 2009; Organización para la Cooperación y el Desarrollo Económico, 2010). ¿Es suficiente con estar alfabetizado en el uso de las tecnologías para ser parte de la Sociedad de la Información y el Conocimiento? ¿Se requieren otras competencias? ¿Qué aporta la alfabetización en información en esta nueva realidad?

Desde la investigación que se viene realizando se tiene la convicción que para ser un ciudadano con capacidades para ejercer sus derechos en la Sociedad de la Información y el Conocimiento es necesario desarrollar nuevas competencias. Si bien el entorno tecnológico ha modificado las formas tradicionales de relacionamiento social, se considera que es una parte del problema. Las tecnologías facilitaron el acceso a un cúmulo inimaginable de información, por lo tanto, se requiere de nuevas capacidades y competencias para su utilización y apropiación.

Ponjuán (2002) explica, que si bien todavía no se ha logrado combatir el analfabetismo tradicional, nos vemos enfrentados a nuevas formas de analfabetismo como es el informacional, que tiene su origen en el desarrollo de las tecnologías de la información para el acceso, el consumo y distribución de la misma. Señala que en este nuevo contexto los individuos requieren de nuevas habilidades y competencias que les permitan acceder a ese cúmulo infinito de información, y desarrollar capacidades para poder utilizarla para su beneficio personal y el de la sociedad en su conjunto.

Por su lado Badwen (2002), expresa que hoy se habla de alfabetización informática, alfabetización en tecnologías de la información y alfabetización electrónica. Todos estos conceptos alcanzan la utilización de las tecnologías, y agrega que se confunde la alfabetización digital con la alfabetización en información, pero ambos son dos conceptos significativamente diferentes.

Miranda (2006) sostiene que la alfabetización en información se refiere a la obtención de habilidades y destrezas que le permiten al individuo ser competente en su relación con la información (selección, acceso, evaluación, apropiación, creación y comunicación).

Belluzzo (2008) hace hincapié en la importancia de la educación en este nuevo contexto, señala que es parte del escenario de transformación y que es la encargada de promover las condiciones de los individuos para lograr el tránsito hacia la Sociedad de la Información y el Conocimiento.

Por lo tanto, se considera que existe cierta complementariedad entre la alfabetización digital y la alfabetización en información. No se puede dar una sin la otra, pero hay una clara línea divisoria, que establece que la primera se centra en el manejo de las herramientas tecnológicas y la segunda en el manejo y apropiación de la información. 
Es en este contexto que se plantea el proyecto de investigación, que pretende responder, entre otras, a las siguientes preguntas: ¿Cuál es el rol que ocupan las Tecnologías de la Información y Comunicación (TIC) en el Plan Ceibal y si son tomadas cómo un medio o un fin en sí mismo? Se aspira a determinar si el Plan Ceibal se presenta como un programa de alfabetización digital o apunta a un proceso más profundo que incluya el desarrollo de destrezas y habilidades específicas para el uso, evaluación y apropiación de la información.

Se pretende contribuir a la formación de individuos críticos, aptos para desarrollar estrategias que promuevan sus habilidades en el uso de la información y potencien sus competencias lectoras en el marco de las políticas de acceso a la Sociedad de la Información y el Conocimiento.

En el proceso de implementación del proyecto se pudo constatar que el Plan Ceibal no ha planteado concretamente acciones hacia la implementación de políticas de alfabetización en información y es por eso que a partir de los estudios realizados proponen unas pautas para la construcción de un modelo específico para dicho Plan.

A continuación se expone la metodología utilizada y la discusión de los resultados preliminares de la investigación que han permitido realizar la formulación primaria de pautas para un Modelo de Alfabetización en Información para el Plan Ceibal. En este sentido es importante destacar que la formulación de las pautas para dicho modelo no se justifica en resultados estadísticos, sino en cuestiones de observación, percepción y exploración de la realidad social estudiada.

\section{Métodos}

La investigación aborda la problemática desde un enfoque metodológico cualitativo que partió de una aproximación empírica para arribar a la construcción de conocimiento teórico (Glaser; Strauss, 1967). Se afilia al paradigma constructivista-interpretativo-emergente, que focaliza su atención en el estudio de la realidad social a partir de la construcción conjunta entre el investigadory el investigado (Bisquerra, 1989). En la investigación son de fundamental importancia las percepciones que las personas trasmiten a través de sus palabras y la observación de su comportamiento (Taylor; Bogdan,
1986). La investigación es de carácter exploratorio y no busca generalizaciones estadísticas. En ella confluyen diferentes visiones sobre la temática, comportamientos, percepciones y teoría hacia la construcción colectiva de una propuesta evaluable.

En cuanto a las técnicas utilizadas durante la investigación, se aplicó una combinación de ellas, tales como: entrevistas a informantes calificados; análisis de la bibliografía sobre la temática y documentos referentes al Plan Ceibal; observación de una experiencia práctica realizada con escolares.

Para el trabajo con informantes calificados, se diseñó una pauta de entrevista abierta que fue aplicada a distintos actores políticos, autoridades de la educación y académicos, cuyos perfiles estaban vinculados a las áreas clave de la investigación. Las preguntas estaban orientadas a recabar información y opiniones sobre los temas centrales de la investigación, a saber: el impacto del Plan Ceibal en el Sistema Educativo Uruguayo, los objetivos y el involucramiento docente; constatar la existencia o no de políticas de alfabetización en información en el marco del Plan Ceibal; saber si los entrevistados distinguían la alfabetización en información de la digital; cuáles deberían ser los elementos de un posible Modelo de Alfabetización en Información para el Plan Ceibal; y si se visualizaba el vínculo entre la alfabetización en información y las competencias lectoras. Se realizaron un total de 12 entrevistas, que fueron analizadas y categorizadas de acuerdo a los criterios establecidos.

Por otro lado, se llevó a cabo la observación del proceso de búsqueda de información que realizan los niños en las escuelas utilizando la computadora. Para guiar el proceso de observación se tomaron como guía de referencia las etapas del Modelo de Alfabetización en Información denominado Gavilán (Eduteka, 2007). Se trabajó con un total de 150 niños, cuyo perfil era pertenecer a sexto año escolar, con una edad promedio de 11-12 años. La finalidad fue exploratoria y no se buscaba generalizaciones al respecto. Se formularon 5 preguntas, con la finalidad de que los niños buscaran en distintas fuentes de información la respuesta a las mismas. Se diseñó una aplicación informática para recoger los datos. Los investigadores acompañaron la experiencia observando directamente el proceso realizado por los niños para la búsqueda y evaluación de la información y 
registrando aquellos aspectos de relevancia para el estudio.

Asimismo, y en forma paralela se trabajó con una segunda pauta dirigida a los maestros. Esta buscaba conocer la forma de trabajo con las computadoras en el aula, específicamente vinculada a la búsqueda, utilización y evaluación de la información. La pauta se aplicó a cinco maestros responsables de los grupos de escolares que participaron de la experiencia práctica de búsqueda de información.

\section{Resultados y Discusión}

\section{La visión de los informantes calificados}

En esta oportunidad se presentan los resultados vinculados a la visión brindada por los entrevistados respecto a la distinción entre alfabetización en información y alfabetización digital y su relación con el fortalecimiento de las competencias lectoras. Por otro lado, se vuelcan las opiniones sobre la necesidad de desarrollar competencias específicas para utilizar la información y en la medida que fuera necesario, que elementos debería incluir un Modelo de Alfabetización en Información.

A partir de las respuestas obtenidas se puede afirmar que existe una comprensión generalizada de lo que implica estar alfabetizado en el uso de la información, y que los entrevistados pudieron distinguir las dos dimensiones: alfabetización en información y digital. Destacaron la necesidad de desarrollar competencias específicas para usar la información sobre todo a partir de la introducción de Internet, y de los riesgos que implica el acceso ilimitado a la información. Señalaron también la necesidad de desarrollar competencias específicas sobre todo en el manejo de información que no está sometida a los controles de calidad habituales del mundo analógico.

Los entrevistados coincidiendo con algunas de las investigaciones realizadas sobre la introducción de las TIC en el aula, señalaron que la tecnología no es suficiente para generar los cambios a nivel educativo o social (Area, 2005, 2010; García-Varcarcel; Tejedo, 2010). Destacaron la necesidad de desarrollar programas específicos que apunten a formar a los estudiantes en el uso de la información, y a los docentes en la incorporación de estos recursos en el aula.

En primera instancia la investigación permitió confirmar que el Plan Ceibal no contempla políticas de alfabetización en información. Este último aspecto fue también compartido por las autoridades del Plan quienes reconocen la importancia de este aspecto, y prevén encaminar acciones en este sentido.

Los entrevistados coincidieron en señalar la necesidad de desarrollar competencias específicas para buscar y utilizar la información. En ese sentido, mencionaron algunos de los aspectos que debería contemplar un Modelo de Alfabetización en Información: búsqueda de información; utilización de distintas fuentes, tanto digitales como análogas; desarrollo de capacidades para seleccionar la información según el interés o necesidad de cada individuo: determinar el origen de la información, reconocer y respetar el derecho de autor; saber citar y hacer una bibliografía; capacidad crítica para evaluar fuentes de información; elementos para procesar la información, (entenderla, captarla, utilizarla con sentido ético) permitiendo un manejo social apropiado; capacidad para distinguir entre distintos tipos de textos.

Otra pregunta que se formula a los entrevistados buscaba responder si la alfabetización en información puede contribuir al fortalecimiento de las competencias lectoras. En general, los entrevistados entienden que hay una relación entre la alfabetización en información y las competencias lectoras. Ubican a las dos como las competencias necesarias para desempeñarse en el siglo XXI tal cual es definido por el Programa Internacional de Evaluación de Estudiantes (PISA) (España, 2006) por autores como Cuevas Cerveró y Garcia-Quismondo (2007) y por la Organización para la Cooperación y el Desarrollo Económico (2010).

Se plantea una última pregunta que busca conocer si la estrategia pedagógica del Plan Ceibal apunta a fortalecer las competencias lectoras. Todos coinciden en señalar la necesidad de que el Plan pueda ser un instrumento que refuerce las competencias lectoras, pero reconocen que recién se está comenzando a trabajar en esta línea. Así, lo explicitan las autoridades del Plan que expresan que se está desarrollando una biblioteca virtual y la incorporación de software específicos que permitan mejorar las competencias lectoras. 


\section{Observación del proceso de búsqueda de información por parte de los niños}

El objetivo que se planteó al observar el proceso de búsqueda de información por parte de los niños fue conocer, cómo a partir de un problema de información concreto, proceden a dar respuesta al mismo. Para ello y como ya fue mencionado se utilizaó como pauta de observación las etapas que define el Modelo Gavilán para el proceso de alfabetización en información (Eduteka, 2007). El mismo establece cuatro etapas por las que debe transitar un individuo cuando está alfabetizado en el uso de la información: 1) definir el problema de información; 2) buscar y evaluar la información; 3) analizar la información; 4) sintetizar la información y utilizarla.

Respecto a nuestra observación se pudo constatar que los niños inician el proceso en el buscador Google (que por defecto presenta la computadora), sin poder distinguir qué es un motor de búsqueda y qué es un recurso de información. Cuando se les solicitó que registraran la dirección del recurso de información que habían utilizado indicaban que había sido Google.

Respecto a las etapas antes mencionadas apenas llegan a la segunda. Se quedan solamente con la búsqueda sin poder hacer una correcta evaluación de la información recuperada. Utilizan el primer recurso que recupera Google, y responden la pregunta con la información que aparece en el resumen del recurso y/o título, sin proceder a abrirlo y a leer la información que contiene el mismo.

Por tanto, se estaría en condiciones de afirmar que los niños no realizan una evaluación de las fuentes de información y tampoco tienen elementos para distinguir entre los diferentes tipos de fuentes. No consultan otros recursos para comparar la información, responden con lo primero que encuentran, prácticamente sincuestionamientos.

Respecto al uso de recursos de información, las observaciones realizadas permiten concluir que hay ciertos sitios que son de consulta habitual en algunas escuelas, y por lo tanto los niños tienen mucha familiaridad con las mismas, como es el caso de Wikipedia. Se pudo observar, que en general no saben usar las guías clasificadas ni los diccionarios bilingües ni otras fuentes de información.
La visión de los maestros

Las respuestas de los maestros permiten confirmar los resultados de algunas de las investigaciones internacionales que concluyen en señalar que la simple dotación de recursos tecnológicos en las escuelas no es suficiente para que los docentes integren las mismas a sus prácticas pedagógicas (Area, 2005, 2010; García Vercarcel; Tejedor, 2010). En el caso del Plan Ceibal, se observa que los maestros realizan las actividades relacionadas con búsqueda y evaluación de la información según su propia formación.

Del análisis de los resultados preliminares, se puede considerar que sería una valiosa contribución la incorporación al Plan Ceibal de un programa de alfabetización en información, dirigido a niños y maestros, que brinde las herramientas para ser un usuario de la información capacitado y calificado, en el marco de un proceso de aprendizaje a lo largo de toda la vida.

\section{Las pautas para el modelo de alfabetización en información}

A partir de los elementos que señalaron los entrevistados, de las carencias constatadas en la experiencia con niños y la visión de los maestros; y teniendo en cuenta las competencias necesarias para desempeñarse en este nuevo contexto según fueron definidas en documentos de referencia y en algunos modelos de alfabetización en información, se definen las competencias que debería contemplar un Modelo de Alfabetización en Información para el Plan Ceibal (Ontorio School Library Association, 1999; Kuhlthau, 2001; Bundy, 2004; Organización para la Cooperación y el Desarrollo Económico, 2010).

TransInformação, Campinas, 25(2):127-133, maio/ago., 2013 
Tabla 1. Competencias a desarrollar en el marco del Modelo de Alfabetización en Información para el Plan Ceibal. Propuesta surgida de la investigación en curso.

\begin{tabular}{ll}
\hline Competencias a desarrollar & $\begin{array}{l}\text { La aplicación del Modelo de Alfabetización en Información } \\
\text { permitirá }\end{array}$ \\
\hline $\begin{array}{l}\text { Gestionar la información eficiente y éticamente a través del correcto uso } \\
\text { de las distintas fuentes. }\end{array}$ & $\begin{array}{l}\text { Aprender a reconocer una necesidad de información. } \\
\text { Iniciación en el proceso básico de la investigación a través del desarrollo } \\
\text { de capacidades en la formulación de problemasy el planteo de preguntas. }\end{array}$ \\
$\begin{array}{l}\text { Conducir búsquedas que le permiten resolver sus problemas de } \\
\text { información y contestar preguntas. }\end{array}$ & $\begin{array}{l}\text { Buscar, seleccionar, analizary evaluar distintos tipos defuentes } \\
\text { de información. }\end{array}$ \\
$\begin{array}{l}\text { Leer y entender los distintos formatos: textos continuos, discontinuos, } \\
\text { hipertextos, multimediales. }\end{array}$ & $\begin{array}{l}\text { Utilizar la información éticamente respetando autorías, } \\
\text { derechos etc. }\end{array}$ \\
\hline
\end{tabular}

Se entiende por competencia a las calificaciones que posee un individuo ya sean físicas, intelectuales y conductuales. Se pueden observar en situaciones reales o de prueba. Aportan valor agregado porque incorporan conocimientos y habilidades en los procesos. Son demostrables. Se relacionan con la ejecución exitosa de una actividad. Se pueden generalizar a más de una actividad y se transfieren a distintos contextos. La formación por competencias no refiere a saber hacer una tarea pura y exclusivamente, sino que abarca, saber el por qué, el para qué, el cómo y necesariamente implica el uso de información ya que se sustenta en tres tipos de actividades: interpretativas, argumentativas y propositivas (Angulo Marcial, 2003).

Se estima que un Modelo de Alfabetización en Información aplicado al Plan Ceibal debería apuntar a desarrollar las siguientes competencias que se pueden visualizar (Tabla 1).

\section{Consideraciones Finales}

En principio se está en condiciones de afirmar que nuestra investigación permitió comprobar que el
Plan Ceibal no contempla políticas de alfabetización en información. Planteado el tema sobre la necesidad de definir un modelo al respecto que complemente el Plan Ceibal dicho Plan y que promueva las capacidades lectoras, fue considerado como fundamental por todos los actores involucrados. La definición de las competencias y capacidades que debe desarrollar un niño escolar que incorpora una computadora al ámbito educativo surgen a partir de las entrevistas realizadas, de las observaciones realizada a los niños en su proceso de búsqueda de información y de las competencias necesarias para desarrollarse en el siglo XXI definidas a nivel internacional.

La propuesta no pretende quedarse en la mera definición teórica de unas pautas sino en avanzar en el desarrollo de un modelo integral que contemple el uso de distintas fuentes de información, su evaluación, el análisis de la información para poder usar la misma de forma ética y responsable. Se pretende conducir este proceso inserto en una evaluación continua de las acciones que se realicen y para ello es necesario trabajar con los actores involucrados y sobre todo con los niños y maestros.

\section{Referencias}

ADMINISTRACIÓN NACIONAL DE EDUCACIÓN PÚBLICA. Evaluación del Plan Ceibal 2010: documento resumen. Montevideo: Plan Ceibal, 2011. Disponible en: <http:// www.anep.edu.uy/anepdata/0000031610.pdf>. Acceso en: 15 abr. 2012.

ANGULO MARCIAL, N. Normas de competencias en información. Biblioteconomia i Documentación, n.11, 2003.
Disponible en: <http://www.ub.es/bid/11angul2.htm>. Acceso en: 15 abr. 2012.

AREA, M. Tecnologías de la información y comunicación en el sistema escolar: una revisión de líneas de investigación. Relieve, v.11, n.1, 2005. Disponible en: <http://www.uv.es/ RELIEVE/v11n1/RELIEVEV11n1_1.htm>. Acceso en: 15 abr. 2012. 
AREA, M. El proceso de integración y uso pedagógico de las TIC en los centros educativos: un estudio de caso. Revista de Educación, v.352, p.77-97, 2010. Disponible en: <http:// www.revistaeducacion.mec.es>. Acceso en: 15 abr. 2012

BADWEN, D. Revisión de conceptos de alfabetización informacional y digital. Anales de Documentación, n.5, p.361408, 2002. Disponible en: <http://revistas.um.es/analesdoc/ article/viewFile/2261/2251>. Acceso en: 15 abr. 2012.

BELLUZZO, R.C.B. Como desenvolver a competencia em informação (Cl): una mediação integrada entre a biblioteca e a escola. CRB-8 Digital, v.1, n.2, p.11-14, 2008. Disponível em: <www.crb8.org.br>. Acesso em: 15 abr. 2012.

BISQUERRA, R. Métodos de investigación educativa. Barcelona: CEAC, 1989.

BUNDY, A. (Ed.). Australian and New Zealand information literacy framework. Adelaide: Australian and New Zealand Institute for Information Literacy, 2004. Available from: <http:// www.library.unisa.edu.au/learn/infolit/Infolit-2ndedition.pdf>. Cited: Apr. 15, 2012.

CUEVAS CEVERÓ, A.; GARCÍA QUISMONDO, M.A.M. La competencia lectora como modelo de alfabetización en información. Anales de Documentación, n.10, p.49-70, 2007. Disponible en: <http://revistas.um.es/analesdoc/article/view/ 1082/1132>. Acceso en: 15 abr. 2012.

EDUTEKA. Modelo Gavilán 2.0: una propuesta para el desarrollo de la competencia para manejar la información. 2007. Disponible en: <http://www.eduteka.org/pdfdir/Modelo Gavilan.pdf>. Acceso en: 15 abr. 2012.

ESPAÑA. Ministerio de Educación y Ciencia. PISA 2006: programa para la evaluación Internacional de alumnos de la OCDE. Madrid: MEC, 2006. Disponible en: <http://www. mepsyd.es/meed/gabipen/documentos/files/informeespañol-pisa-2006.pdf>. Acceso en: 15 abr. 2012.

GARCIA-DESMONDO, M.A.M. Evolución conceptual de la alfabetización en información a partir de la alfabetización múltiple en su perspectiva educativa y bibliotecaria. Investigación Bibliotecológica, v.23, n.47, p.129-160, 2009. Disponible en: <http://www.ejournal.unam.mx/ibi/vol23-47/ IBI002304706.pdf>. Acceso en: 15 abr. 2012.

GARCIA-VARCARCEL, A.;TEJEDOR, F.J. Evaluación del proceso de innovación escolar basados en el uso de las TIC desarrollados en la Comunidad de Castilla y León. Revista de Educación, v.352, p.125-147, 2010. Disponible en: <http:// www.revistaeducacion.mec.es/re352/re352_06.pdf>. Acceso en: 15 abr. 2012.

GLASER, B.G.; STRAUSS, A.L. The discovery of grounded theory. Chicago: Aldine, 1967.
KUHLTHAU, C. El rediseño de las bibliotecas escolares en la era informática: roles fundamentales para el aprendizaje basado en investigación. Eduteka, 2001. Disponible en: <http:// www.eduteka.org/profeinvitad.php3?ProflnvID $=0007$ > . Acceso en: 15 abr. 2012.

MARTINEZ, A.L.; ALONSO, S.; DÍAZD. Monitoreoyevaluación de impacto social del Plan Ceibal. Montevideo: Plan Ceibal, 2009. Disponible en: <http://www.ceibal.org.uy/docs/presentacion _impacto_social 221209.pdf>. Acceso en: 15 abr. 2012.

MIRANDA, S. Como as necesidades de informação podem se relacionar com as competências informacionais. Ciência da Informação, v.35, n.3, 2006. Disponível em: <http:// www.scielo.br/pdf/ci/v35n3/v35n3a10.pdf>. Acesso em: 15 abr. 2012.

ONTARIOSCHOOL LIBRARY ASSOCIATION. Information studies kindergarten to grade 12: curriculum for schools and school library information centre. Toronto: The Ontario School Library Association, 1999. Available from: <http://www.accessola. com/data/6/rec_docs/113_Information_Studies.pdf >.Cited: Apr. 15, 2012.

ORGANIZACIÓN PARA LA COOPERACIÓN Y EL DESARROLLO ECONÓMICOS. Habilidades y competencias en el siglo XXI para los aprendices del nuevo milenio en los países de la OCDE. Paris: Instituto de Tecnologías Educativas, 2010. Disponible en: <http:// recursostic.educacion.es/blogs/europa/media/blogs/europa/ informes/Habilidades_y_competencias_siglo 21_OCDE.pdf $>$. Acceso en: 15 abr. 2012.

PITTALUGA, L. ¿Contribuye el Plan Ceibal a cerrar la brecha digital en Uruguay: algunos indicios? In: SEMINARIO INTERNACIONAL POLITICAS SOCIALES Y LA SOCIEDAD DE LA INFORMACIÓN, BRECHAS, OPORTUNIDADES Y DERECHOS, 2010, Santiago de Chile. Anale electrónicos... Santiago de Chile: Naciones Unidas, 2010. Disponible en: <http:// www.cepal.org/dds/noticias/paginas/2/41012/plan-ceibaluruguay-lucia-pittaluga.pdf>. Acceso en: 15 abr. 2012.

PLAN CEIBAL. Objetivos. 2010. Disponible en: <http://www. ceibal.org.uy/index.php?option=com_content\&view= article\&id=44\&ltemid=56>. Acceso en: 15 abr. 2012.

PONJUAN, G. Papel de la colaboración entre líderes de varios sectores para la creación de una cultura informacional. Praga: UNESCO, 2002.

TAYLOR, S.; BOGDAN, R. Introducción a los métodos cualitativos de investigación: la búsqueda de significados. Buenos Aires: Paidós, 1986.

WARSCHAUER, M.; AMES, M. Can one laptop per child save the world's poor? Journal of International Affairs, v.64, n.1, p.33-51, 2010. 